\title{
Sweeping jet from a fluidic oscillator in crossflow
}

\author{
Florian Ostermann, Philipp Godbersen, Rene Woszidlo, C. Navid Nayeri, and C. Oliver Paschereit \\ Institut für Strömungsmechanik und Technische Akustik, HFI, Technische Universität Berlin, \\ 10623 Berlin, Germany
}

(Received 8 August 2017; published 29 September 2017)

\begin{abstract}
This paper is associated with a video winner of a 2016 APS/DFD Milton van Dyke Award for work presented at the DFD Gallery of Fluid Motion. The original video is available from the Gallery of Fluid Motion, https://doi.org/10.1103/APS.DFD.2016.GFM.V0076
\end{abstract}

DOI: 10.1103/PhysRevFluids.2.090512

Jets in crossflow are a fundamental flow scenario relevant for various technical applications. Recent studies have indicated promising improvements for several applications by using spatially oscillating jets (i.e., sweeping jets) generated by fluidic oscillators. These are devices that are able to emit a sweeping jet without requiring any moving parts. This advantage makes them attractive for flow control applications. Several studies have proven their effectiveness for mixing enhancement, reduction of drag and noise, and separation control [1]. However, the reasons for their effectiveness remains widely unknown due to the lack of fundamental knowledge on the oscillators themselves and on the interaction between the sweeping jet and a crossflow. Ostermann et al. described the time-resolved internal mechanisms inside a fluidic oscillator $[2,3]$ and investigated the interaction of a sweeping jet with a crossflow [4,5]. The video presented here provides a time-resolved, threedimensional visualization of the experimentally acquired flow field of a sweeping jet interacting with a crossflow.

The experiments are conducted in a wind tunnel where the fluidic oscillator is installed inside a splitter plate. The oscillation plane spanned by the sweeping jet is perpendicular to the crossflow direction. The velocity ratio between jet and crossflow is three and the oscillation frequency is $67 \mathrm{~Hz}$. A traversable stereoscopic particle image velocimetry (PIV) system is employed for acquiring the flow field plane-by-plane. A pressure signal provides a temporal correlation between the various PIV planes, which enables phase-averaging to yield the three-dimensional and time-resolved flow field for one oscillation period [6]. More information on the setup are provided in Ref. [4].

After describing the setup, the video displays the velocity magnitude, which shows the sweeping movement of the jet. It also indicates that the jet is being bent into the direction of the crossflow. The streak volume of the jet (Fig. 1) reveals more detailed flow features. The streak volume is obtained by tracing virtual particles in the flow field through time. Due to its visual similarity to ink-based visualizations, it enables a more intuitive interpretation of the flow field and exposes dominant flow features. Two counter-rotating vortices oriented in streamwise direction are very prominent. They form alternatingly downstream of the jet. An instantaneous cross-section through the flow field visualizes their sense of rotation (Fig. 2). In-plane velocity vectors infer that their sense of rotation is opposite to that of the counter-rotating vortex pair formed downstream of a steady jet in crossflow. Therefore, fluid motion toward the wall is induced in between these vortices. Based on inviscid vortex dynamics, this sense of rotation keeps the vortices close to the wall over a longer downstream distance, thereby affecting a greater area. The presence of such dominant streamwise vortices and their sense of rotation is suspected to be one aspect for the high efficacy of sweeping jets in flow

Published by the American Physical Society under the terms of the Creative Commons Attribution 4.0 International license. Further distribution of this work must maintain attribution to the author(s) and the published article's title, journal citation, and DOI. 


\section{FLORIAN OSTERMANN et al.}

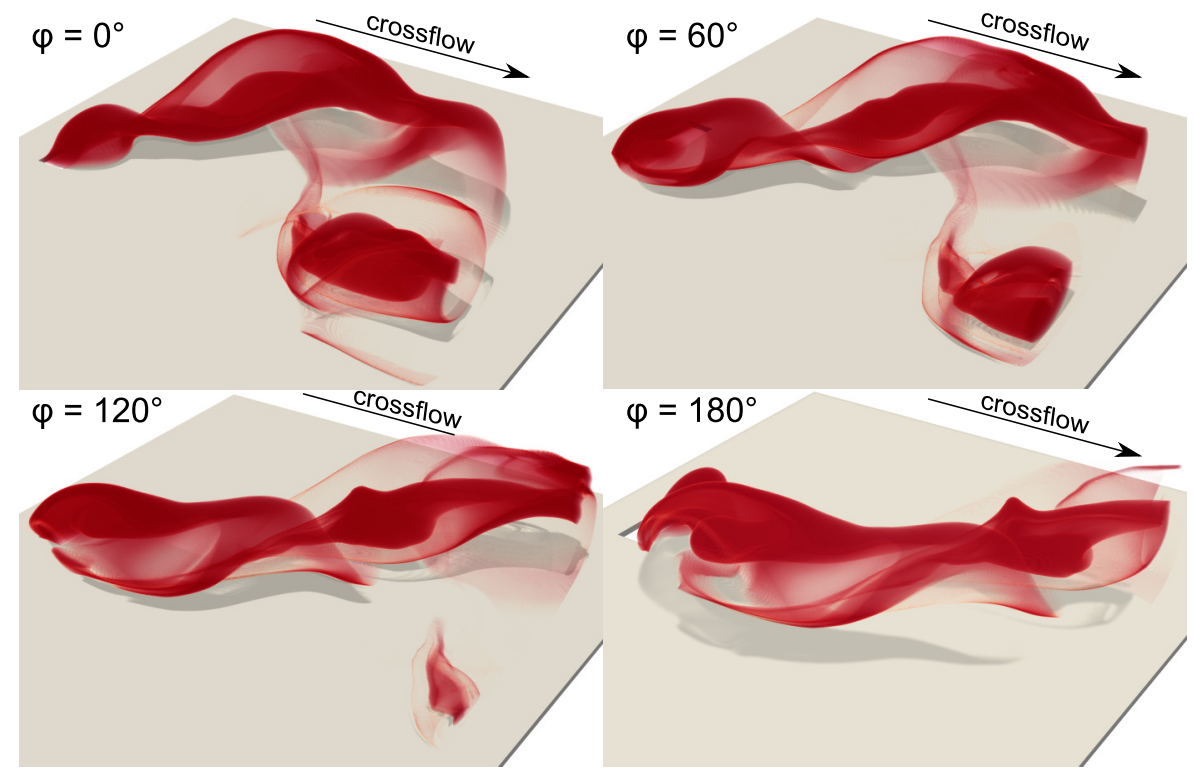

FIG. 1. A streak volume illustrating the flow field of a spatially oscillating jet in crossflow for various time steps. The video associated with these images is available at https://doi.org/10.1103/ APS.DFD.2016.GFM.V0076

control applications. At the end of the video, the finite-time Lyapunov exponent (FTLE) is shown. It does not only visualize the jet itself but also flow structures of the crossflow (e.g., flat plate boundary layer).

The video highlights the spatial and temporal flow field complexity of a spatially oscillating jet interacting with a crossflow. It provides a qualitative overview of dominant flow features inside the flow field by using various visualization techniques. It is noteworthy that the highlighted flow structures are linked to Strouhal number based on oscillation frequency and change with this parameter [5].

The authors gratefully acknowledge the funding from the Deutsche Forschungsgemeinschaft (DFG Project No. PA 920/34-1).

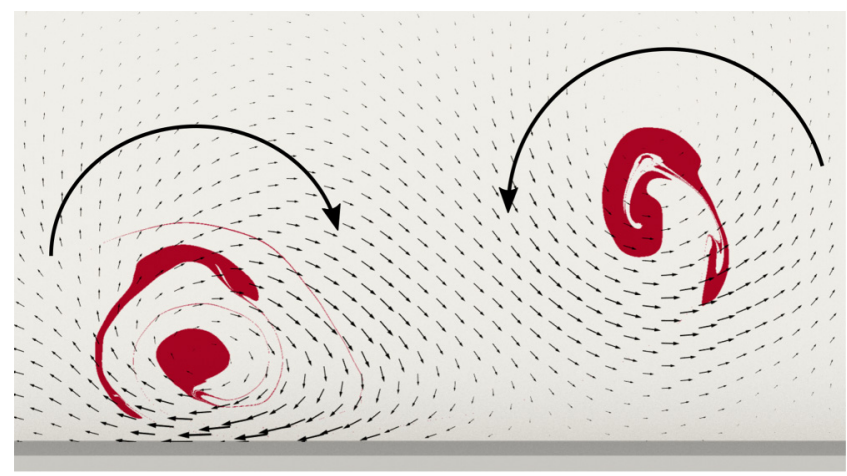

FIG. 2. A cross-section at the downstream end of the wall. 


\section{SWEEPING JET FROM A FLUIDIC OSCILLATOR IN . . .}

[1] J. W. Gregory and M. N. Tomac, A review of fluidic oscillator development, in Proceedings of the 43rd AIAA Fluid Dynamics Conference, San Diego, CA (American Institute of Aeronautics and Astronautics, Reston, VA, 2013).

[2] R. Woszidlo, F. Ostermann, C. N. Nayeri, and C. O. Paschereit, The time-resolved natural flow field of a fluidic oscillator, Exp. Fluids 56, 125 (2015).

[3] M. Sieber, F. Ostermann, R. Woszidlo, K. Oberleithner, and C. O. Paschereit, Lagrangian coherent structures in the flow field of a fluidic oscillator, Phys. Rev. Fluids 1, 050509 (2016).

[4] F. Ostermann, R. Woszidlo, C. Nayeri, and C. O. Paschereit, The time-resolved flow field of a jet emitted by a fluidic oscillator into a crossflow, in Proceedings of the 54th AIAA Aerospace Sciences Meeting, San Diego, CA (American Institute of Aeronautics and Astronautics, Reston, VA, 2016).

[5] F. Ostermann, R. Woszidlo, C. N. Nayeri, and C. O. Paschereit, Effect of velocity ratio on the flow field of a spatially oscillating jet in crossflow, in Proceedings of the 55th AIAA Aerospace Sciences Meeting, Grapevine, TX (American Institute of Aeronautics and Astronautics, Reston, VA, 2017).

[6] F. Ostermann, R. Woszidlo, C. N. Nayeri, and C. O. Paschereit, Phase-averaging methods for the natural flowfield of a fluidic oscillator, AIAA J. 53, 2359 (2015). 